\title{
Low-Footprint Optical Interconnect on an SOI Chip Through Heterogeneous Integration of InP-Based Microdisk Lasers and Microdetectors
}

Joris Van Campenhout, Pietro R. A. Binetti, Pedro Rojo Romeo, Philippe Regreny, Christian Seassal, Xaveer J. M. Leijtens, Tjibbe de Vries, Yoki Siang Oei, Rene P. J. van Veldhoven, Richard Nötzel, Léa Di Cioccio, Jean-Marc Fedeli, Meint K. Smit, Dries Van Thourhout, and Roel Baets

\begin{abstract}
We present a proof-of-principle demonstration of a low-footprint optical interconnect on a silicon-on-insulator (SOI) chip. The optical link consists of a heterogeneously integrated, InP-based microdisk laser (MDL) and microdetector, coupled to a common SOI wire waveguide. Applying an electrical current to the MDL resulted in a detector current up to $1 \mu \mathrm{A}$.
\end{abstract}

Index Terms-Heterogeneous integration, microdisk laser (MDL), optical interconnect, silicon photonics.

\section{INTRODUCTION}

$\mathbf{S}$ ILICON photonics has emerged as a potentially low-cost technology for applications such as short-range optical interconnect and optical sensing with high sensitivity. A major drawback of the silicon platform, however, is the lack of efficient, compact, and integrated light sources and detectors, due to the indirect bandgap of silicon. Heterogeneous integration with InP-based materials is a promising route to extend the reach of silicon photonics [1], [2]. In this letter, we present first results on a silicon-on-insulator (SOI)-integrated, low-footprint optical link, fabricated through direct bonding technology. We discuss the measured static link performance and give an outlook for future improvement.

\footnotetext{
Manuscript received December 01, 2008; revised January 17, 2009. Current version published March 27, 2009. This work was supported by the E.U. through the IST-PICMOS project.

J. Van Campenhout was with Ghent University - IMEC, INTEC, B-9000 Gent, Belgium. He is now with IBM Research, Yorktown Heights, NY 10598 USA (e-mail: jvancam@us.ibm.com).

P. R. A. Binetti, X. J. M. Leijtens, T. de Vries, Y. S. Oei, P. J. van Veldhoven, R. Nötzel, and M. K. Smit are with the COBRA Research Institute, Technische Universiteit Eindhoven, 5600 MB Eindhoven, The Netherlands (e-mail: p.r.a.binetti@tue.nl; x.j.m.leijtens@tue.nl; t.d.vries@tue.nl; y.s.oei@tue.nl; p.j. veldhoven@tue.nl; r.noetzel@tue.nl; m.k.smit@tue.nl).

P. R. Romeo, P. Regreny, and C. Seassal are with INL, Université de Lyon, Institut des Nanotechnologies de Lyon INL-UMR5270, CNRS, F-69134 Ecully Cedex, France (e-mail: Pedro.Rojo-Romeo@ec-lyon.fr; Philippe.Regreny@eclyon.fr; Christian.Seassal@ec-lyon.fr).

L. Di Cioccio and J.-M. Fedeli are with the CEA-LETI, 38054 Grenoble, France (e-mail: lea.dicioccio@cea.fr; jean-marc.fedeli@cea.fr).

D. Van Thourhout and R. Baets are with Ghent University-IMEC, INTEC, B-9000 Gent, Belgium (e-mail: dries.vanthourhout@intec.ugent.be; roel.baets@intec.ugent.be).

Color versions of one or more of the figures in this letter are available online at http://ieeexplore.ieee.org.

Digital Object Identifier 10.1109/LPT.2009.2014391
}

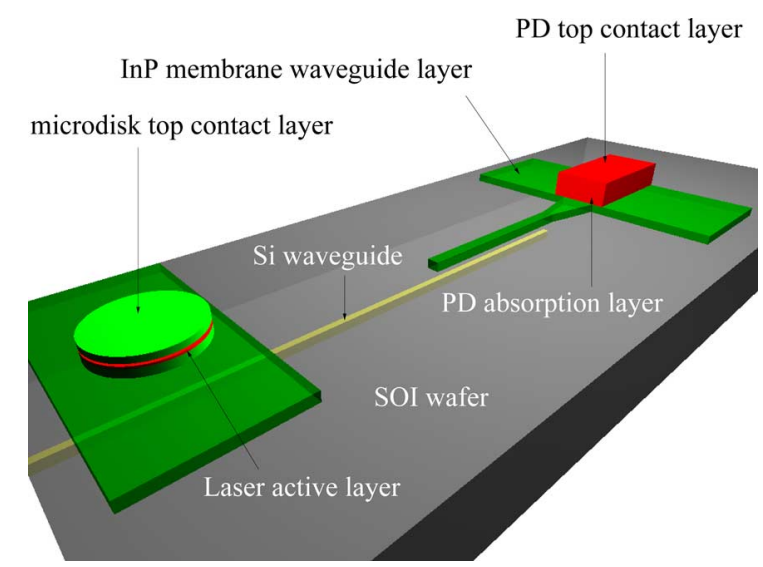

Fig. 1. Schematic diagram of a photonic link on an SOI substrate: The electrically pumped MDL (left) launches an optical signal into the $\mathrm{Si}$ waveguide. Light is collected by the detector structure (right).

\section{DESIGN}

In our optical link, transverse-electric polarized light from an electrically driven InP-based microdisk laser (MDL) is coupled evanescently into the Si waveguide in the interconnection layer. The optical power is carried by the $\mathrm{Si}$ waveguide $\left(500 \times 220 \mathrm{~nm}^{2}\right.$ in cross section) over the link and is coupled into a photodetector (PD) structure by means of an InP membrane coupler: A schematic drawing of the MDL-to-PD point-to-point link is shown in Fig. 1.

The MDL is built around a InAsP multiquantum-well active layer in a nonintentionally doped (n.i.d.) InGaAsP structure core, which is sandwiched by an n-type contact layer and tunnel-junction-based p-type contact [1].

The PD structure is implemented as an n.i.d. InGaAs absorption layer sandwiched between a highly p-doped InGaAs contact layer and a highly n-doped InP layer, which is also used for realizing a membrane waveguide acting as a coupling structure. The microdisk and the detector mesa footprints are $50 \mu \mathrm{m}^{2}$. The laser and the detector layer stack were chosen to reach a trade-off between the ease of integration and device performance. More details about the detector structure design can be found in [3].

\section{FABRICATION}

Two InP-based 2-in wafers with the MDL and the PD layer stacks were epitaxially grown by solid-source molecular-beam 


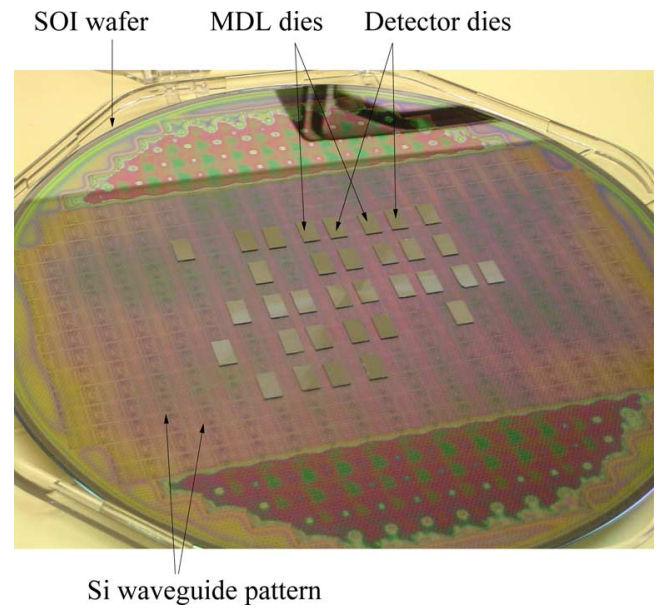

Fig. 2. Laser and detector InP-based dies bonded on a 200-mm SOI wafer.

epitaxy and by metal-organic vapour-phase epitaxy, respectively. The InP wafers were diced in $9 \times 4.5 \mathrm{~mm}^{2}$ pieces, which were then bonded upside down on an SOI wafer (see Fig. 2), in which the Si waveguide pattern had been defined [4]. Fiber grating couplers (FGCs) [5] were etched into the Si photonic waveguide layer to enable monitoring the optical power in the $\mathrm{Si}$ waveguide during the characterisation of the link. $\mathrm{A} \mathrm{SiO}_{2}$ layer was deposited on top of the SOI and InP wafers to provide a silica-to-silica interface for the molecular bonding [6]. After bonding, the InP die substrate was removed by a combination of mechanical grinding and wet-chemical etching, and laser and PD patterns were defined by e-beam lithography and transferred to a 150 -nm-thick $\mathrm{SiO}_{2}$ hard mask. The SOI wafer was subsequently sawn into samples containing both MDL and PD dies. We proceeded with separate processing steps for the MDLs and the PDs, by covering one die with thick protective photoresist while processing the other. The $\mathrm{SiO}_{2}$ hard mask was used to dry-etch the microdisks in the laser die, while in the PD die it masked the dry-etch of the membrane waveguides and of the PD bottom contact areas, which share the same n-InP layer. The silica mask on top of the detectors was then dry-etched away and the mesas were wet-chemically etched. Finally, some common processing steps were performed to complete the chip. A benzocyclobutene layer was spin-coated to planarize the chip surface and provide electrical isolation. Device topand side-contact windows were opened by a combination of $\mathrm{O}_{2}$-plasma etch and dry etching, and metal was evaporated and patterned by lift off. Top and bottom PD contacts were metallized with a Ti-Pt-Au layer stack, as well as the bottom MDL contacts, while a Ti-Au layer stack was used for MDL top contacts. Fig. 3 shows the mask layout and a picture of the fabricated devices, taken with an optical microscope before the chip metallization.

\section{MeAsurement Results}

The static performance of the integrated optical link was tested at room temperature by applying a variable current to the MDL and recording the corresponding PD current. The MDL in the link of interest was $500 \mathrm{~nm}$ thick and had a diameter of

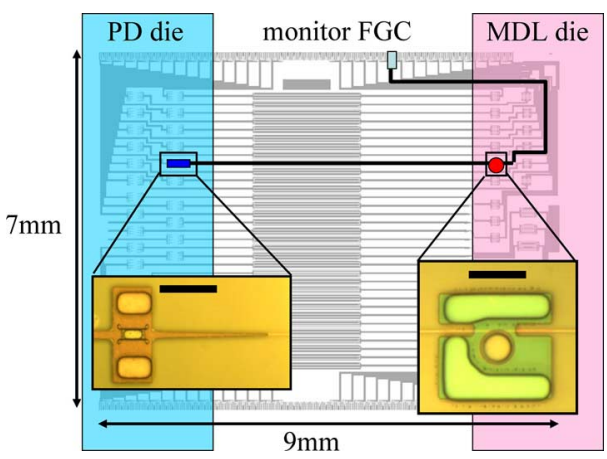

Fig. 3. Layout of the test chip, showing the position of the bonded laser and detector die, the Si photonic waveguide pattern (background) and the FGCs at the edges of the chip. Pictures of an MDL and a PD taken with an optical microscope before the chip metallization are shown in the inset (scale bar is $10 \mu \mathrm{m}$ ).
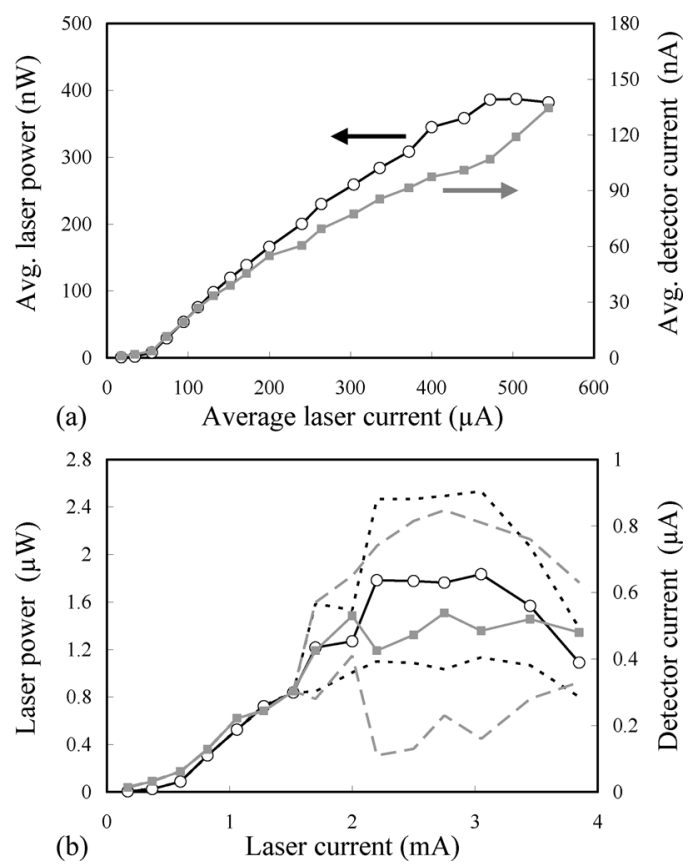

Fig. 4. (a) Optical power arriving at the FGC (black) and detector current (grey), measured as function of laser drive current for the pulsed and (b) the continuous-wave experiment. The measured optical power (detector current) fluctuated between the values indicated by the dotted (dashed) lines.

$7.5 \mu \mathrm{m}$. The dimensions of the detector were $30 \times 5 \mu \mathrm{m}^{2}$. The length of the link was $7 \mathrm{~mm}$.

In a first measurement, a pulsed current drive signal (80-ns pulses, $1-\mu$ s period) was applied to the MDL in order to avoid excessive self heating [1], and the average detector current was recorded as function of the laser current. The PD was kept unbiased at $0 \mathrm{~V}$ throughout the experiment, as the detectors on this particular sample suffered from relatively large dark currents (several $\mu \mathrm{A}$ ) at elevated reverse bias level, most probably due to unexpected erosion of the top p-contact layer, which occurred during the mesa wet-etching step. At the same time, the average optical power in the SOI waveguide was monitored using the FGC at the end of the SOI wire opposite to the one terminated by the detector (Fig. 3). Both curves can be found in Fig. 4(a). The threshold of the laser is reached at an average laser current of $55 \mu \mathrm{A}$, or equivalently, a peak laser current of 


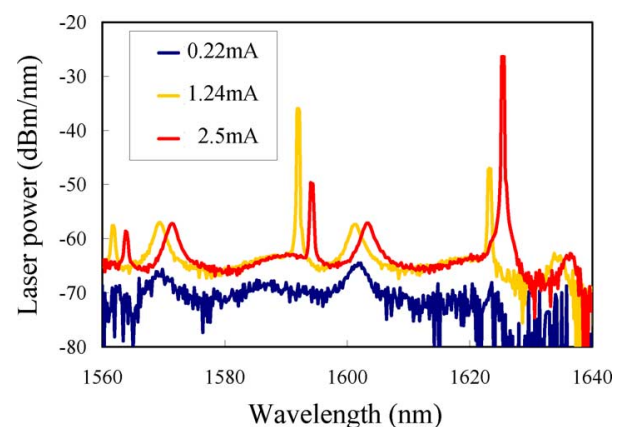

Fig. 5. MDL spectra, recorded at the monitor FGC for three continuous-wave bias currents (normalized for FGC efficiency).

$700 \mu \mathrm{A}$, as apparent from the kink in both curves. The waveguide-coupled laser slope efficiency, evaluated just before the FGC, is about $1.8 \mu \mathrm{W} / \mathrm{mA}$, accounting for an FGC efficiency of $25 \%$. The measured average detector current varies almost linearly with the measured optical power, especially for average laser currents below $200 \mu \mathrm{A}$. We can estimate the PD responsivity to be $0.33 \pm 0.04 \mathrm{~A} / \mathrm{W}$, if we assume that the MDL emits equal amounts of power in both directions of the SOI waveguide and that the propagation losses for both the MDL-FGC and MDL-PD light paths are equal. This responsivity agrees reasonably well with the $0.45 \mathrm{~A} / \mathrm{W}$ reported in [3]. At the maximum average (peak) laser current of $545 \mu \mathrm{A}(6.8 \mathrm{~mA})$, the recorded average (peak) detector current is as high as $140 \mathrm{nA}(1.75 \mu \mathrm{A})$.

In a second measurement, the MDL was driven in continuous-wave regime [see Fig. 4(b)]. The lasing threshold is about $600 \mu \mathrm{A}$ and the slope efficiency just above threshold is $1.6 \mu \mathrm{W} / \mathrm{mA}$. For drive currents below $1.5 \mathrm{~mA}$, the measured detector current shows a clear correlation with the monitor laser power, with an estimated detector responsivity of $0.35 \pm 0.07 \mathrm{~A} / \mathrm{W}$, which is consistent with the pulsed experiment. For laser currents above $1.5 \mathrm{~mA}$, the dominant lasing wavelength red-shifts to a lower order whispering-gallery mode at $1625 \mathrm{~nm}$ due to self heating, as is shown in Fig. 5. For these higher drive currents, the optical power detected through the monitor FGC was found to fluctuate strongly with drive current. Fig. 4(b) shows the envelope of the recorded values as well as the averaged value. The self heating is also obvious from the thermal roll-over of the average light output-current $(L-I)$ curve. The detector current was also found to vary drastically with changing laser drive current. Furthermore, it was found that a drop in laser power at the monitor grating coupler coincides with a peak in detector current and vice versa. Therefore, the laser clearly has a preferential emission direction, which changes with drive current. This is a result of mode competition between the clockwise and counterclockwise laser modes. This mode competition is strongly affected by reflection feedback caused by the FGC, which is stronger at $1625 \mathrm{~nm}(-10 \mathrm{~dB})$ than at $1590 \mathrm{~nm}(-15 \mathrm{~dB})$ [5]. Since the phase of this feedback is highly sensitive to the emission wavelength, the preferential output direction can vary abruptly with drive current as the temperature and emission wavelength of the laser cavity drift due to self heating. We speculate that this effect also affects the dominant output direction at high drive currents in the pulsed experiment, causing the laser to emit slightly more power toward the FGC. It is clear that further measures need to be taken to control the directionality of the MDL output, for instance by adding a functional reflector in the SOI waveguide.

\section{DISCUSSION AND CONCLUSION}

The current-to-current differential efficiency of the present link is fairly poor at about $0.27 \mu \mathrm{A} / \mathrm{mA}(0.027 \%)$. The biggest opportunity for improving efficiency is in enhancing the differential efficiency of the MDL. We already have experimentally demonstrated efficiencies as high as $30 \mu \mathrm{W} / \mathrm{mA}$ [1], and a theoretical study shows that slope efficiencies as high as $260 \mu \mathrm{W} / \mathrm{mA}$ are feasible [7]. By optimizing the detector design, we believe its responsivity could be increased to $1 \mathrm{~A} / \mathrm{W}$. Therefore, a differential link efficiency as high as $10 \%$ seems within reach. The maximum direct modulation bandwidth of the MDL was experimentally assessed elsewhere to be about $5 \mathrm{~Gb} / \mathrm{s}$ [8]. A fully optimized disk laser is expected to support data rates well above $10 \mathrm{~Gb} / \mathrm{s}$. The frequency response of the detector was measured to be rather flat up to $20 \mathrm{GHz}$ [3]. Calculations lead to an expected bandwidth of around $30 \mathrm{GHz}$.

In conclusion, we have presented a proof-of-principle demonstration of a low-footprint optical interconnect on an SOI chip, fabricated through heterogeneous integration of InP-based MDLs and microdetectors. While the demonstrated (static) link efficiency is rather low $(0.027 \%)$, optimizing the individual components is expected to yield link efficiencies up to $10 \%$, and a data rate of $10 \mathrm{~Gb} / \mathrm{s}$ and above.

\section{REFERENCES}

[1] J. Van Campenhout, P. Rojo Romeo, D. Van Thourhout, C. Seassal, P. Regreny, L. Di Cioccio, J.-M. Fedeli, C. Lagahe, and R. Baets, "Electrically pumped InP-based microdisk lasers integrated with a nanophotonic silicon-on-insulator waveguide circuit," Opt. Express, vol. 15, no. 11, pp. 6744-6749, 2007.

[2] A. W. Fang, R. Jones, H. Park, O. Cohen, O. Raday, M. J. Paniccia, and J. E. Bowers, "Integrated AlGaInAs-silicon evanescent racetrack laser and photodetector," Opt. Express, vol. 15, no. 5, pp. 2315-2322, 2007.

[3] P. Binetti, X. Leijtens, T. de Vries, Y. Oei, O. Raz, L. Di Cioccio, J.-M. Fedeli, C. Lagahe, R. Orobtchouk, J. Van Campenhout, D. Van Thourhout, P. van Veldhoven, R. Nötzel, and M. Smit, "Indium posphide based membrane photodetector for optical interconnects on silicon," in Proc. LEOS Annual, Newport Beach, CA, Nov. 2008, pp. 302-303, Paper TuT4.

[4] W. Bogaerts, R. Baets, P. Dumon, V. Wiaux, S. Beckx, D. Taillaert, B. Luyssaert, J. Van Campenhout, P. Bienstman, and D. Van Thourhout, "Nanophotonic waveguides in silicon-on-insulator fabricated with CMOS technology," J. Lightw. Technol., vol. 23, no. 1, pp. 401-412, Jan. 2005.

[5] D. Taillaert, W. Bogaerts, P. Bienstman, T. Krauss, P. V. Daele, I. Moerman, S. Verstuyft, K. D. Mesel, and R. Baets, "An out-of-plane grating coupler for efficient butt-coupling between compact planar waveguides and single-mode fibers," IEEE J. Quantum Electron., vol. 38, no. 7, pp. 949-955, Jul. 2002.

[6] L. D. Cioccio, "New result obtained at LETI on 3D heterostructures," in Abstracts 210th Meeting Electrochemical Society, Cancun, Mexico, Oct. 29-Nov. 3, 2006, p. 602, Abstract 1649.

[7] J. Van Campenhout, P. Rojo-Romeo, D. Van Thourhout, C. Seassal, P. Regreny, L. Di Cioccio, J. M. Fedeli, and R. Baets, "Design and optimization of electrically injected InP-based microdisk lasers integrated on and coupled to a SOI waveguide circuit," J. Lightw. Technol., vol. 26, no. 1, pp. 52-63, Jan. 1, 2008.

[8] L. Liu, J. Van Campenhout, G. Roelkens, D. Van Thourhout, P. Rojo-Romeo, P. Regreny, C. Seassal, J.-M. Fedeli, and R. Baets, "Ultralow-power all-optical wavelength conversion in a silicon-on-insulator waveguide based on a heterogeneously integrated III-V microdisk laser," Appl. Phys. Lett., vol. 93, p. 061107, 2008. 